\title{
FAR INFRARED GALAXIES IN AKARI'S EYE
}

\author{
K. Malek $^{1,2}$, A. Pollo ${ }^{3,4}$, T. T. Takeuchi ${ }^{1}$, E. Giovannoli ${ }^{5}$, V. Buat ${ }^{5}$, \\ D. Burgarella ${ }^{5}$, and M. Malkan ${ }^{6}$ \\ ${ }^{1}$ Department of Particle and Astrophysical Science, Nagoya University, Furo-cha, Chikusa-ku, \\ Nagoya 464-8602, Japan \\ ${ }^{2}$ Center for Theoretical Physics of the Polish Academy of Science, Al. Lotnikow 32/46, 02-668 Warsaw, Poland \\ ${ }^{3}$ National Centre for Nuclear Research, ul. Andrzeja Sołtana 7, 05-400 Otwock, Świerk, Poland \\ ${ }^{4}$ Astronomical Observatory of the Jagiellonian University, ul. Orla 171, 30-244, Kraków, Poland \\ ${ }^{5}$ Laboratoire d'Astrophysique de Marseille, OAMP, Université Aix-Marseille, CNRS, 38 rue Frederic Joliot-Curie, \\ 13388 Marseille, cedex 13, France \\ ${ }^{6}$ Department of Physics and Astronomy, Unversity of California, Los Angeles, CA 90024-1547, USA \\ E-mail: malek.kasia@nagoya-u.jp \\ (Received July 01, 2012; Accepted August 01, 2012)
}

\begin{abstract}
We present the results of Spectral Energy Distribution (SED) fitting of far-infrared galaxies detected in the AKARI Deep Field-South (ADF-S) Survey and discuss their physical properties. Additionally, we perform a comparison between photometric redshifts estimated using only optical and both optical and infrared data. We conclude that our sample consists mostly of nearby galaxies rich in dust and young stars. We observe an improvement in the estimation of photometric redshifts when the IR data are included, comparing to a standard approach based mainly on the optical to UV photometry.
\end{abstract}

Key words: surveys; galaxy: fundamental parameters; galaxies: evolution; infrared: galaxies

\section{INTRODUCTION}

AKARI, the Japanese satellite dedicated to infrared (IR) astronomy (Murakami et al., 2007), was launched with the main purpose of creating an all-sky survey, but it also performed two deep surveys around the ecliptic poles. Our analysis is based on one of these deep surveys: the AKARI Deep Field South (ADF-S). This field, characterized by the lowest Galactic cirrus emission and located near the South Ecliptic Pole, allows for the best far-infrared (FIR) extragalactic image of the Universe. Using the Far-Infrared Surveyor (FIS: Kawada et al., 2007) onboard AKARI, it was possible to create a FIR map of the ADF-S field which covers approximately 12 square degrees. This survey was done in four photometric bands: $60 \mu \mathrm{m}, 90 \mu \mathrm{m}, 140 \mu \mathrm{m}$, and $160 \mu \mathrm{m}$. 2263 infrared sources were detected down to $\sim 20 \mathrm{mJy}$ in the $90 \mu \mathrm{m}$ band. The first analysis of this sample in terms of the nature and properties for 1,000 ADF-S objects brighter than 0.301 Jy in the 90 $\mu \mathrm{m}$ band was presented by Małek et al. (2010).

\section{DATA}

The main aim of our work is to build a galaxy sample with high quality fluxes from the ultraviolet (UV) to the FIR using the ADF-S sample. We use the ADF-S multiwavelength catalogue (Małek et al., 2010), based on the 1,000 ADF-S brightest sources in the $90 \mu \mathrm{m}$ band, which contains 545 galaxies identified by finding counterparts in other databases. Additional measurements, mostly from WISE (Wright et al., 2010) and GALEX (Dale et al., 2007), and additional information 
TABLE 1.

The Main Measurements Included in Our Database

\begin{tabular}{cccc}
\hline \hline Survey & Band & Wavelength & $\#_{\text {all }}$ \\
\hline \hline GALEX & FUV & $0.1 \mu m$ & 78 \\
GALEX & NUV & $0.2 \mu m$ & 98 \\
HIPASS & $B_{j}$ & $0.4 \mu m$ & 401 \\
2MASS & $\mathrm{J}$ & $1.2 \mu \mathrm{m}$ & 204 \\
2MASS & $\mathrm{H}$ & $1.7 \mu \mathrm{m}$ & 204 \\
2MASS & $\mathrm{Ks}$ & $2.2 \mu \mathrm{m}$ & 204 \\
WISE & $\mathrm{W} 1$ & $3.4 \mu \mathrm{m}$ & 280 \\
WISE & $\mathrm{W} 2$ & $4.6 \mu \mathrm{m}$ & 280 \\
WISE & $\mathrm{W} 3$ & $12 \mu \mathrm{m}$ & 280 \\
MIPS & $24 \mu \mathrm{m}$ & $23.7 \mu \mathrm{m}$ & 100 \\
AKARI & N60 & $65 \mu \mathrm{m}$ & 116 \\
AKARI WIDE-S & $90 \mu \mathrm{m}$ & 1000 \\
IRAS & Band-4 & $100 \mu \mathrm{m}$ & 30 \\
AKARIWIDE-L & $140 \mu \mathrm{m}$ & 65 \\
AKARI & N160 & $160 \mu m$ & 9 \\
\hline
\end{tabular}

from public databases $\left(\mathrm{SIMBAD}^{1}, \mathrm{NED}^{2}\right.$, and $\mathrm{IRSA}^{3}$ ) were used in our analysis. In Table 1, we summarize the main data used for our analysis.

\subsection{Redshift Information}

Spectroscopic redshifts $\left(z_{\text {spec }}\right)$ are available for 129 galaxies among the 545 galaxies identified in Matek et al. (2010). Some of them were collected from public databases (NED and SIMBAD). Additionally, we use redshift measurements of Sedgwick et al. (2011).

In our sample, 416 sources are identified in public catalogs as galaxies, with photometric information but no redshift.

In order to analyze the properties of all the identified galaxies, we decided to estimate the photometric redshifts $\left(z_{\text {phot }}\right)$ of the whole sample. We used two codes: Photometric Analysis for Redshift Estimate (Le PHARE ${ }^{4}$; Arnouts et al., 1999; Ilbert et al., 2006), and the Code Investigating GALaxy Emission (CIGALE) SED fitting program (Noll et al., 2009).

CIGALE is not developed as a tool for estimation of $z_{\text {phot }}$ but since it uses a large number of models covering the whole spectrum including IR, it is expected to

\footnotetext{
1 http://simbad.u-strasbg.fr/simbad/.

2 http://ned.ipac.caltech.edu/.

3 http://irsa.ipac.caltech.edu/.

4 http://www.cfht.hawaii.edu/ arnouts/LEPHARE/lephare. html.
}

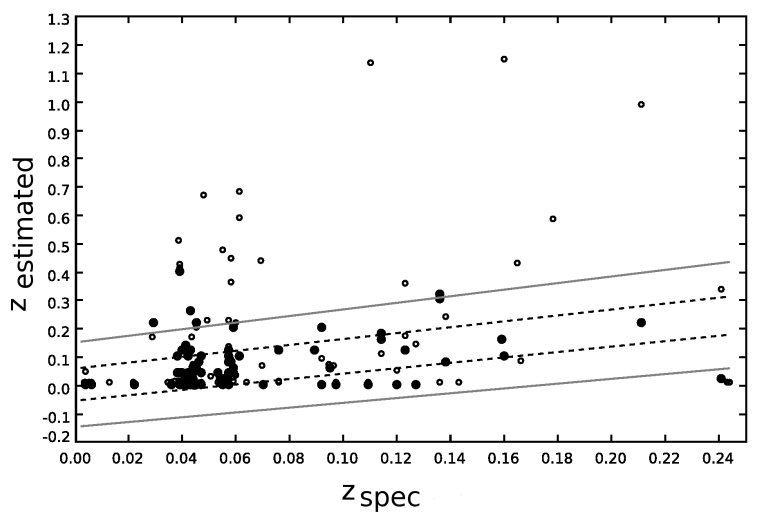

Fig. 1. $z_{\text {phot }}$ (open circles) and $z_{\text {CIGALE }}$ (full circles) versus spectroscopic redshifts for a test sample of 95 galaxies with known $z_{\text {spec }}$. A region of catastrophic errors is defined as $\left|z_{\text {spec }}-z_{\text {phot }}\right| /\left(1+z_{\text {spec }}\right)>0.15$ (Ilbert et al., 2006), and marked as a black solid line. Dashed-dotted lines correspond to the $z_{\text {phot }}=z_{\text {spec }} \pm 0.055 \cdot\left(1+z_{\text {spec }}\right)$. Eight galaxies without $z_{\text {phot }}$ calculated successfully are not included at this plot.

provide better $z_{\text {phot }}$ than software using mainly optical to UV data.

We performed a test on the sample of 95 galaxies with known $z_{\text {spec }}$ and at least 6 photometric measurements on the whole spectral range (see Fig. 1) and found that less than $10 \%$ of $z_{\text {CIGALE }}$ may be biased by catastrophic errors, while for the same sample Le PHARE was not able to determine redshifts for $8 \%$ sources, and in additional $12 \%$ cases the estimated values were affected by catastrophic errors. Results plotted in Fig. 1 show that the difference between estimated and spectroscopic redshifts, in the case of catastrophic errors, is higher for $z_{\text {phot }}$ than $z_{\text {CIGALE }}$. Consequently, we decided to use $z_{\text {CIGALE }}$ for sources without known spectroscopic redshift for the subsequent analysis.

\section{SED FITTING}

\subsection{Sample Selection}

To study the physical parameters of ADF-S sources we selected galaxies with known $z_{\text {spec }}$ or $z_{\text {CIGALE }}$ and with the highest quality photometry available. The main selection criteria were to have redshift information, and at least six measurements across the whole galaxy spectral range. As a result, we use 129 galaxies with $z_{\text {spec }}$ and 128 galaxies with $z_{\text {CIGALE }}$. All available photometric measurements were then used for the SED fitting 


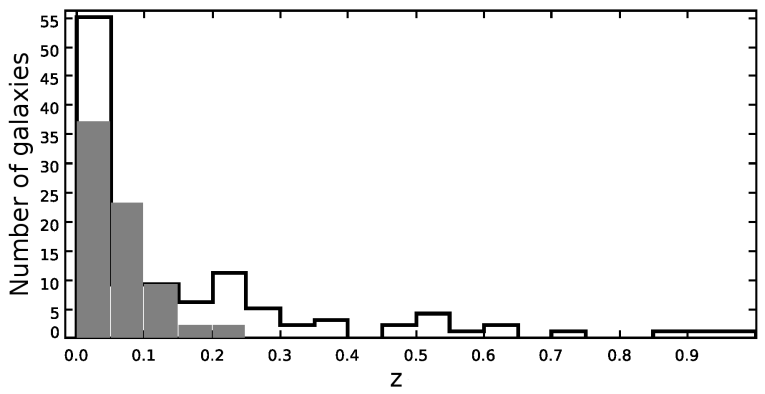

Fig. 2. The redshift distribution of the 186 galaxies used for SED fitting with $\chi^{2}$ value (from the Bayesian analysis) lower than 4. Full histogram represents galaxies with known spectroscopic redshifts. Distribution of galaxies with estimated $z_{\text {CIGALE }}$ redshifts is shown as a open histogram.

\section{with CIGALE.}

CIGALE uses models describing emission from a galaxy in the wavelength range from FUV to FIR (see Noll et al., 2009).

A mock catalog was constructed to check the reliability of the parameter estimation by CIGALE, following the procedure described by Giovannoli et al. (2011). The comparison between the results from mock and real catalogs demonstrates that CIGALE provides a very good estimation of physical parameters like stellar mass $\left(M_{\text {star }}\right)$, star formation rate (SFR), 4,000 break (D4000, defined as the ratio of the flux in the red continuum to that in the blue continuum), dust attenuation in the $\mathrm{V}\left(A_{\mathrm{V}}\right)$ and FUV $\left(A_{\mathrm{FUV}}\right)$ bands, dust attenuation in the $\mathrm{V}$ band for the young stellar population $\left(A_{\mathrm{v}, \mathrm{ySP}}\right)$, and bolometric and dust luminosity $\left(L_{\mathrm{bol}}, L_{\mathrm{dust}}\right)$, with values of the linear Pearson moment correlation coefficient higher than 0.8 .

\section{RESULTS}

We restrict further analysis to the SEDs with a minimum value of $\chi^{2}$ lower than four. With this condition, 73 galaxies with $z_{\text {spec }}$ and 113 galaxies with estimated $z_{\text {CIGALE }}$ were successfully used for SEDs fitting.

Redshift distribution for our sample is shown on Fig. 2. The mean value of $z_{\text {spec }}$ is equal to $0.067 \pm$ 0.043 , while its median value is equal to 0.047 . The distribution of $z_{\text {CIGALE }}$ is characterized by mean value $0.159 \pm 0.208$ but with a long tail reaching $z=1$. This high $z$ tail is not necessarily due to catastrophic

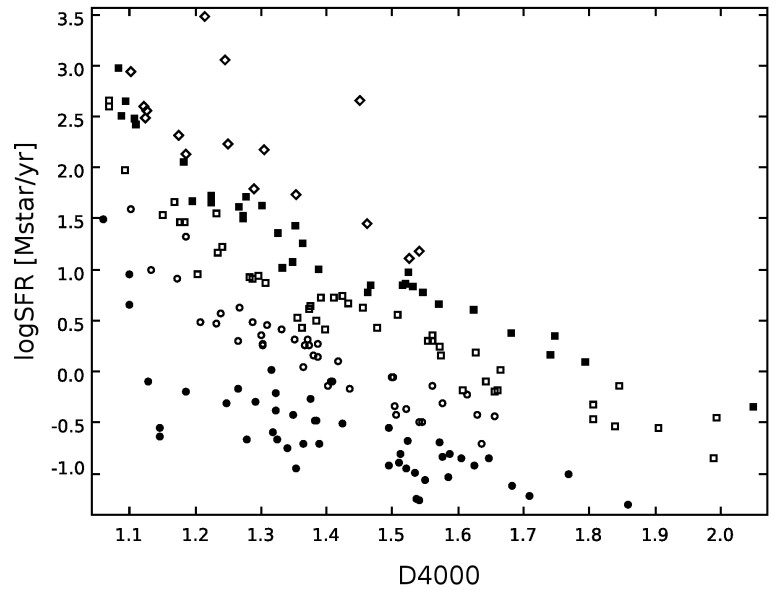

Fig. 3. The relation between the D4000 parameter and $\operatorname{logSFR}$. Open circles represent galaxies with $\log M_{\text {star }}<10$, full circles are sources with $10<\log M_{\text {star }}<10.5$ Open squares correspond to galaxies with $10.5<\log M_{\text {star }}<11$. Galaxies with $11<\log M_{\text {star }}<11.5$ are marked as full squares. Sources with $\log M_{\text {star }}$ higher than 11.5 are plotted as open diamonds.

errors: it can also be real, since our sample of $z_{\text {spec }}$ is strongly biased towards low redshifts by the selection made by the observers (Sedgwick et al., 2011). The median value of $z_{\text {CIGALE }}$ is closer to those of $z_{\mathrm{spec}}$ and equal to 0.06. It suggests that our sample consists mainly of nearby galaxies, luminous in FIR. We found that galaxies in our sample are typically very massive, with median value of $M_{\text {star }}=3.28 \times 10^{10} M_{\odot}$. Moreover, the analyzed sample is rather luminous, $L_{\mathrm{bol}}=$ $5.15 \times 10^{10} L_{\odot}$, also in the dust-dominated part of the spectrum $\left(L_{\text {dust }}=1.56 \times 10^{10} L_{\odot}\right)$. The median value of the star formation rate parameter, SFR, is equal to $2.22 M_{\odot} \mathrm{yr}^{-1}$. The median value of $A_{\mathrm{V}}$ parameter, describing dust effective attenuation for stellar population at wavelength equal $5,500 \AA$ is $0.47 \mathrm{mag}$, and median value for attenuation in FUV filter (at 1,500 $\left.\AA, A_{\mathrm{FUV}}\right)$ is $1.87 \mathrm{mag}$. The parameter $A_{\mathrm{V}, \mathrm{ySP}}$, which describes V-band attenuation for young stellar population model, spreads through almost the entire range of input parameter from 0.1 to 2.25 , with median value $0.97 \mathrm{mag}$.

The D4000 may provide information about the age of the stellar population. The break in spectra occurs on the grounds of the accumulation of a large number of spectral lines in a narrow wavelength region and it 
is strongly connected to ionized metals, wherefore the D4000 break is strong for old galaxies, rich in metals, and much weaker for galaxies with young stellar populations (Kauffmann et al., 2003). Kauffmann et al. (2003) found that the distribution of stellar mass shows a clear division between galaxies dominated by old and more recent star formation, which are discriminated by their D4000 parameter. D4000 1.3 describes young galaxies, and old, elliptical galaxies are concentrated for typical D4000 value of 1.85. Most objects in our sample $(64.52 \%)$ have low values of D4000 $(<1.5)$. Galaxies with depth of Balmer break lower than 1.3 comprise $31.72 \%$. It suggests that our sample consists of active, dust-rich galaxies, dominated by young stellar population.

We found that D4000 is strongly connected to SFR (see Fig. 3). Galaxies characterized by low factor of D4000 frequently have a very high instantaneous star formation rate. This dependency in our sample is also related to the stellar mass and redshift - the massive galaxies in our sample have higher redshifts and lower D4000.

\section{ACKNOWLEDGEMENTS}

This work is based on observation with AKARI a JAXA project with the participation of ESA. This research has made use of the SIMBAD and NED databases. $\mathrm{KM}$ and $\mathrm{AP}$ were financed by the research grant of the Polish National Science Centre N N203 512938 . This research was partially supported by the project POLISH-SWISS ASTRO PROJECT cofinanced by a grant from Switzerland through the Swiss Contribution to the enlarged European Union. KM has been supported from the Japan Society for the Promotion of Science (JSPS) Postdoctoral Fellowship for Foreign Researchers, P11802. TTT has been supported by the Grant-in-Aid for the Scientific Research Fund (20740105, 23340046, and 24111707) and for the Global COE Program Request for Fundamental Principles in the Universe: from Particles to the Solar System and the Cosmos commissioned by the Ministry of Education, Culture, Sports, Science and Technology (MEXT) of Japan. VB and DB have been supported by the Centre National des Etudes Spatiales (CNES) and the Programme National Galaxies (PNG).

\section{REFERENCES}

Arnouts, S., Cristiani, S., Moscardini, L., et al., 1999,
Measuring and Modelling the Redshift Evolution of Clustering: the Hubble Deep Field North, MNRAS, 310, 540

Buat, V., Giovannoli, E., Takeuchi, T. T., Heinis, S., Yuan, F.-T., Burgarella, D., Noll, S., \& IglesiasPáramo, J., 2011, Spectral Energy Distributions of an AKARI-SDSS-GALEX Sample of Galaxies, A\&A, 529, A22

Dale, D. A., et al., 2005, Infrared Spectral Energy Distributions of Nearby Galaxies, ApJ, 633, 857

Giovannoli, E., Buat, V., et al., 2011, Population Synthesis Modelling of Luminous Infrared Galaxies at Intermediate Redshift, A\&A, 525, A150

Ilbert, O., Arnouts, S., McCracken, H. J., et al., 2006, Accurate Photometric Redshifts for the CFHT Legacy Survey Calibrated Using the VIMOS VLT Deep Survey, A\&A, 457, 841

Kauffmann, G., Heckman, T. M., White, et al., 2003, Stellar Masses and Star Formation Histories for $10^{5}$ Galaxies from the Sloan Digital Sky Survey, MNRAS, 341, 33

Kawada, M., Baba, H., Barthel, P. D., et al., 2007, The Far-Infrared Surveyor (FIS) for AKARI, PASJ, 59, 389

Małek, K., Pollo, A., Takeuchi, T. T., et al., 2010, Star Forming Galaxies in the AKARI Deep Field South: Identifications and Spectral Energy Distributions, A\&A, 514, A11

Murakami, H., Baba, H., Barthel, P. D., et al., 2007, The Infrared Astronomical Mission AKARI, PASJ, 59, 369

Noll, S., Burgarella, D., Giovannoli, E., et al., 2009, Analysis of Galaxy Spectral Energy Distributions from Far-UV to Far-IR with CIGALE: Studying a Sings Test Sample, A\&A, 507, 1793

Sedgwick, C., Serjeant, S., Pearson, C., et al., 2011, Far-Infrared Luminosity Function of Local StarForming Galaxies in the AKARI Deep FieldSouth, MNRAS, 416, 1862

Wright, E. L., Eisenhardt, P. R. M., et al., 2010, The Wide-field Infrared Survey Explorer (WISE): Mission Description and Initial On-orbit Performance, AJ, 140, 1868 